\title{
Characteristics of Symptomatic Basilar Artery Stenosis Using High- Resolution Magnetic Resonance Imaging in Ischemic Stroke Patients
}

\author{
Hyun Goo Kang ${ }^{1}$, Chan-Hyuk Lee ${ }^{1}$, Byoung-Soo Shin ${ }^{1}$, Gyung Ho Chung ${ }^{2}$ and Hyo Sung Kwak ${ }^{2}$
}

Hyun Goo Kang and Chan-Hyuk Lee contributed equally to this work.

\begin{abstract}
${ }^{1}$ Department of Neurology and Research Institute of Clinical Medicine of Jeonbuk National University-Biomedical Research Institute of Jeonbuk National University Hospital, Jeonju, Republic of Korea

${ }^{2}$ Department of Radiology and Research Institute of Clinical Medicine of Jeonbuk National University-Biomedical Research Institute of Jeonbuk National University Hospital, Jeonju, Republic of Korea
\end{abstract}

Aim: Arterial narrowing associated with the progression of atherosclerosis leads to serious conditions such as stroke, coronary artery disease, or even death. High-resolution magnetic resonance imaging (HR-MRI) is better for detecting arterial wall status and discriminating tissue characteristics than conventional imaging. We used HR-MRI to investigate the frequency of patients with basilar artery (BA) stenosis observed distinctively on routine angiography and identify the clinical features associated with this imaging. We analyzed the nature of the vessel wall causing the basal artery stenosis by HR-MRI, and related clinical factors.

Methods: Patients with BA stenosis underwent HR-MRI. The association between atherosclerosis (with or without intraplaque hemorrhage [IPH]) and dissection was analyzed. High signal intensity within a BA plaque on magnetization-prepared rapid acquisition with gradient echo was defined as an area with a signal intensity $>200 \%$ that of the adjacent muscle.

Results: Fifteen patients were diagnosed with BA dissection on HR-MRI. IPH was identified in 14 patients. Patients with BA plaque with IPH were older and had higher prevalence of hypertension and hyperlipidemia than the other patients. The frequencies of alcohol drinking and number of current smokers were higher in the dissection group than in the other groups. Hyperlipidemia was identified as an influencing factor for IPH development in atherosclerotic plaque. Young age was identified as the influencing factor for the occurrence of BA dissection.

Conclusions: The etiology of stenosis or occlusion was unclear until the development of HR-MRI. With HRMRI, stroke etiology is better understood, and factors affecting each etiology can be identified. Further studies that clarify the etiology of posterior circulation stroke are required.

Key words: Atherosclerosis, Basilar artery, Dissection, Magnetic resonance imaging, Stroke

\section{Introduction}

The basilar artery (BA) is a very small intracranial artery (approximately $3 \mathrm{~mm}$ in diameter) that provides blood to the brain stem. Posterior circulation ischemic stroke accounts for only approximately 30\% of all ischemic stroke cases, but it is associated with higher mortality and morbidity rates than anterior circulation ischemic stroke ${ }^{1)}$. The major cause of BA stenosis is atherosclerosis with or without intraplaque hemorrhage (IPH). However, BA dissection accounts

Address for correspondence: Hyo Sung Kwak, Department of Radiology and Research Institute of Clinical Medicine of Jeonbuk National University-Biomedical Research Institute of Jeonbuk National University Hospital, 20 Geonji-ro, Deokjin-gu, Jeonju 54907, Republic of Korea E-mail: kwak8140@jbnu.ac.kr

Received: May 28, 2020 Accepted for publication: September 28, 2020

Copyright@2020 Japan Atherosclerosis Society

This article is distributed under the terms of the latest version of CC BY-NC-SA defined by the Creative Commons Attribution License. 
for some cases of BA stenosis.

$\mathrm{IPH}$ is observed in atherosclerotic plaques, suggesting the presence of unstable plaques, which are commonly found in the majority of advanced plaques. When neovascularization occurs in a plaque, plaque progression is induced and rupture can occur ${ }^{2)}$. When plaque progression or rupture occurs, ischemic stroke may occur, as BA stenosis can be aggravated by increased plaque burden or emboli can advance to distal portions of the BA. Arterial dissection is a rare condition observed in $2.5 \%$ of the total cases of stroke $^{3)}$. However, it is one of the important etiologies for stroke in young patients and is observed in $10 \%-$ $25 \%$ of young stroke patients. It can be caused by physical trauma or genetic connective tissue diseases, and frequently occurs in the carotid or vertebral arteries with high mobility. Arterial dissection was difficult to diagnose in the past, but it is becoming easier to establish the diagnosis of dissection as vascular imaging technology continues to develop.

IPH and arterial dissection have different etiologies, and consequently, they require different treatment strategies. Therefore, distinguishing the difference between IPH and dissection before treating patients with suspected BA stenosis is considered important. Both atherosclerotic plaques and arterial dissection may be observed as simple stenosis during time-of-flight (TOF) magnetic resonance (MR) angiography (MRA). On the contrary, high-resolution MR imaging (HR-MRI), which is frequently used, is useful for differentiating various blood vessel conditions and is helpful in tracking and observing the changes within the blood vessel walls ${ }^{4)}$. Therefore, it is expected that the changes caused by various etiologies such as atherosclerosis or dissection can be distinguished.

HR-MRI studies associated with arterial stenosis or dissection have mostly focused on the extracranial arteries. However, East Asians, including Koreans and Chinese, experience stenosis or dissection of intracranial arteries significantly more frequently than they do of extracranial arteries. Studies on stenosis and dissection are urgently required ${ }^{5,6}$. We categorized BA stenosis according to the nature of the vessel wall by using HR-MRI, and identified the influencing factors related to the etiologies of stenosis and dissection.

\section{Materials and Methods}

\section{Study Population}

This study was conducted with the approval of the local institutional review board, and each patient provided informed consent before their images were obtained. Consecutive 117 patients diagnosed with
BA stenosis using TOF-MRA between January 2015 and December 2018 were selected. Standard brain MRI and MRA were performed in all patients to detect any other lesions. HR-MRI was also performed to evaluate BA stenosis within 2 weeks after the initial MR examination. We enrolled symptomatic patients who showed evidence of an ischemic stroke or transient ischemic attack within the stenotic area of the BA and a hyperintense signal on diffusionweighted imaging with a related decreased signal on the clear diffusion coefficient map within the preceding week. We excluded patients who met any of the following conditions from the analysis: (1) coexistent unilateral or bilateral vertebral artery stenosis or luminal irregularity of $>50 \%$ on MRA or HR-MRI; (2) $<50 \%$ BA stenosis or complete BA occlusion found on MRA and HR-MRI; (3) other vasculopathies such as inflammatory arteritis or Moyamoya disease; (4) presence of atrial fibrillation; (5) HR-MRI performed for a previously confirmed follow-up stenotic lesion; (6) dissecting aneurysm; or (7) clinical contraindications to MRI. We defined BA dissection as wall thickening with low signal intensity due to an intimal flap on black-blood (BB) T2-weighted imaging and high signal intensity due to intimal flap on TOF-MRA ${ }^{7)}$.

A total of 117 patients underwent HR-MRI during the study period. Forty-three patients were excluded from the analysis; they included 25 with $<50 \%$ BA stenosis on axial images of HR-MRI, 12 with coexistent unilateral or bilateral vertebral artery stenosis or luminal irregularity of $>50 \%, 2$ with other vasculopathies, and 4 undergoing HR-MRI on follow-up. As a result, this study analyzed 74 patients.

We reviewed patient's cardiovascular risk factors including hypertension, diabetes mellitus, and hyperlipidemia. Detailed guideline for hyperlipidemia is as follows: We diagnosed patients with hyperlipidemia when patients had already been diagnosed with hyperlipidemia and had been taking statin drugs or when the results of the lipid profile, which was determined at the time of admission, met one of the following criteria: 1) triglyceride $>200 \mathrm{mg} /$ $\mathrm{dL}$; 2) high-density lipoprotein cholesterol $<40 \mathrm{mg} /$ $\mathrm{dL}$; and 3) low-density lipoprotein (LDL) cholesterol satisfying the criteria specified in the 2018 Guideline on the Management of Blood Cholesterol published by the American College of Cardiology ${ }^{8)}$.

\section{Magnetic Resonance Imaging (MRI)}

The MRI protocol was similar to that of a previous study [2]. A 3T MRI scanner (Achieva; Philips Medical Systems, Amsterdam, Netherlands) with a 16-channel head coil was used for MRI. All 
study subjects initially underwent conventional brain MRI, including three-dimensional (3D) TOF-MRA. TOF-MRA of the axial plane was performed for each subject, and data were reconstructed using an online postprocessing tool dedicated to constructing blood vessel architectures.

The HR-MRI protocol comprises five MR scans: BB T1-weighted, BB T2-weighted, TOF axial, magnetization-prepared rapid acquisition with gradient echo (MPRAGE), and contrast-enhanced BB T1-weighted imaging. T1-weighted imaging was obtained using a two-dimensional turbo spin-echo sequence under the following conditions: repetition time/echo time, $800 / 10 \mathrm{~ms}$; field of view, $140 \times 140$ $\mathrm{mm}$; matrix size, $140 \times 150$; slice thickness, $2.0 \mathrm{~mm}$; echo train length, 10; and number of excitations, 2 . T2-weighted HR-MRI scans also used a turbo spinecho sequence with the following conditions: repetition time/echo time, $3100 / 80 \mathrm{~ms}$; field of view, $140 \times 140 \mathrm{~mm}$; matrix size, $140 \times 140$; slice thickness, $2.0 \mathrm{~mm}$; echo train length, 20; and number of excitations, 2. The imaging parameters for the TOFMRA scan were as follows: repetition time/echo time, $18 / 3.8 \mathrm{~ms}$; flip angle, $16^{\circ}$; field of view, $140 \times 140$ $\mathrm{mm}$; matrix size, $312 \times 165$; slice thickness, $1.0 \mathrm{~mm}$; echo train length, 1 , and number of excitations, 3 . For the 3D MPRAGE sequencing, the segment was acquired using sequence repetition time, inversion preparation time, and the phase encoding order obtained from the MPRAGE sequences, and it was adjusted to optimally identify IPH as hyperintense. The image parameter settings were as follows: repetition time/echo time/inversion time, 8.8/5.3/304 $\mathrm{ms}$; flip angle, $15^{\circ}$; echo train length, 32; field of view, $140 \times 140 \mathrm{~mm}$; and matrix, $216 \times 198$. The BB technique based on preregional $80-\mathrm{mm}$-thick saturation pulses, which saturates incoming arterial flow, was used for all scans. Gadodiamide $(0.1 \mathrm{mmol} /$ kg body weight; Dotarem; Guerbet, Aulnay-sous-Bois, France) was administered intravenously to all patients before contrast-enhanced BB T1-weighted imaging. Contrast-enhanced BB T1-weighted imaging was performed approximately 5 minutes after the contrast injection.

The longitudinal coverage of each artery was 22-24 mm. Each scan lasted 3-4 minutes. The total scan time was within 25 and 30 minutes, and patients remained in the MR machine for 35-45 minutes.

\section{Image Analysis}

This study examined the presence of BA plaques and BA arterial dissection across all samples using HR-MRI. Two neuroradiologists (G.H.C. and HSK) and two neurologists (HGK and BSS) performed all of these procedures together. The image analysis methods employed in this study were similar to those in a previous study protocol ${ }^{2,7)}$.

This study defined plaque as a thickening of the focal wall compared to the image slices obtained from beneath or above the focal wall, as identified on T2and T1-weighted imaging. When high signal intensity was detected within the BA plaques, they were considered as IPH-positive on MPRAGE sequence. High signal intensities are signal intensities of the adjacent muscles greater than $200 \%$ on MPRAGE $^{9,10)}$. The associations among BA plaque with IPH, BA plaque without IPH, and BA dissection were analyzed. All slices of a BA plaque on HR-MRI were re-examined by two experienced image readers who were not informed about the clinical details and did not know the results of the diffusion-weighted images. When their interpretations differed, consensus interpretation was used for the final analysis.

Interpreters searched for direct evidence of $\mathrm{BA}$ dissection, such as a mural hematoma and/or dissection flaps. This study defined a mural hematoma as the detection of eccentric, intermediate-to-high signal intensity of the arterial wall according to hemorrhagic age on T1-weighted imaging and/or MPRAGE $^{6}$. A layer crossing the arterial lumen was defined as a dissection flap ${ }^{6}$. The layer could either be a low signal intensity layer within a high signal intensity hematoma or an enhanced layer on contrastenhanced T1-weighted images ${ }^{11,12)}$. To distinguish a dissection flap from an inflow artifact, which is usually found at the center of the lumen, we considered a layer that extended to the arterial sidewall on any serial image as a dissection flap ${ }^{6}$.

\section{Statistical Analyses}

The demographics, risk factors, and proportion of patients with BA plaque, with or without IPH, and arterial BA dissection were assessed. Pearson's chisquared test, Fisher's exact test, Students $t$-test, and Wilcoxon rank-sum test were used for categorical or continuous variables as considered appropriate. Univariate and multivariate analysis models were used to investigate the factors associated with $\mathrm{BA}$ plaque with IPH and BA dissection. To avoid variable selection caused by spurious correlations, only variables showing a potential association $(p<0.1)$ in the univariate analysis were included in the multivariate logistic regression model. A two-sided $p$ value of $<0.05$ was considered statistically significant. All statistical analyses were performed using the Statistical Package for the Social Sciences version 21 (International Business Machines Corp., Armonk, NY, USA). 

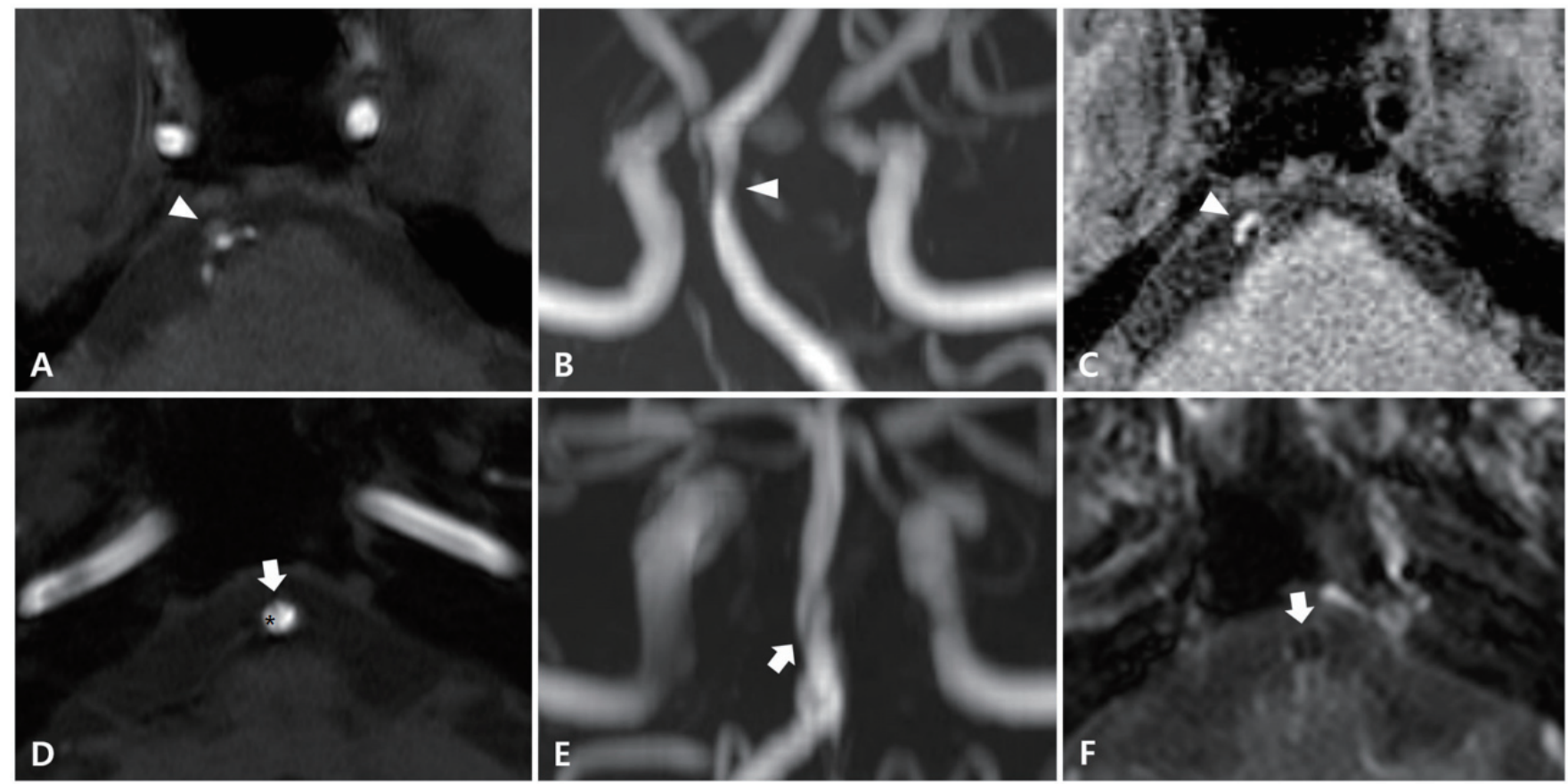

Fig. 1. Differences between atherosclerosis and dissection of basilar artery (BA) on high-resolution magnetic resonance (MR) imaging A-C: Atherosclerotic plaque with intraplaque hemorrhage (arrow head) A: time-of-flight (TOF) axial imaging in the BAs indicates an eccentric plaque with low signal intensity. B: TOF-MR angiography reveals moderate stenosis in the mid-BAs. C: magnetization-prepared rapid acquisition with gradient echo imaging shows an eccentric lesion with high signal intensity in the same area. D-F: Arterial dissection (arrow) D: TOF axial imaging in the BAs indicates the false lumen (asterisk) which shows similar signal intensity compared to true lumen (asterisk). E: TOF-MR angiography reveals intimal flap in the mid-BAs. F: contrast-enhanced black-blood T1-weighted imaging shows intimal flap with low signal intensity.

Table 1. Baseline characteristics of basilar artery atherosclerosis (with or without intraplaque hemorrhage) and dissection

\begin{tabular}{|c|c|c|c|c|}
\hline & \multicolumn{2}{|c|}{ BA atherosclerotic plaque $(n=59)$} & \multirow{2}{*}{$\begin{array}{l}\text { BA dissection } \\
\quad(n=15)\end{array}$} & \multirow{2}{*}{$p$ value } \\
\hline & Without IPH $(n=45)$ & With IPH $(n=14)$ & & \\
\hline Age (years) & $63.7 \pm 12.9$ & $70.8 \pm 8.1$ & $51.1 \pm 14.5$ & $<0.001$ \\
\hline Male & $32(71.1)$ & $6(42.9)$ & $11(73.3)$ & 0.120 \\
\hline Hypertension & $33(73.3)$ & $13(92.9)$ & $7(46.7)$ & 0.021 \\
\hline Diabetes mellitus & $11(24.4)$ & $3(21.4)$ & $2(13.3)$ & 0.664 \\
\hline Hyperlipidemia & $16(35.6)$ & $9(64.3)$ & $2(13.3)$ & 0.017 \\
\hline Alcohol drinking & $15(33.3)$ & $3(21.4)$ & $10(66.7)$ & 0.026 \\
\hline Smoking & $15(33.3)$ & $2(14.3)$ & $8(53.3)$ & 0.084 \\
\hline
\end{tabular}

Results are expressed as number (\%) or mean \pm standard deviation

$\mathrm{BA}$, basilar artery; IPH, intraplaque hemorrhage

\section{Results}

The mean age of all 74 subjects was $62.5 \pm 13.9$ years, and $66.2 \%$ of them were male. Forty-five patients $(60.8 \%)$ had $\mathrm{BA}$ plaque without IPH, 14 (18.9\%) had BA plaque with IPH, and 15 (20.3\%) had BA dissection (Fig. 1). Out of the 59 patients with BA plaque (without IPH, 45; with IPH, 14), 10
(16.9\%) had a distinctive necrotic core.

This study compared the characteristics of patients with BA plaque with or without IPH and BA dissection (Table 1). The mean age of the patients with BA dissection was significantly younger, whereas patients with BA plaque with IPH were the oldest (51.1 \pm 14.5 years old for BA dissection, 63.7 \pm 12.9 years old for BA plaque without IPH, and 70.8 \pm 8.1

\section{Advance Publication Journal of Atherosclerosis and Thrombosis}


Table 2. Univariate and multivariate analyses of the parameters associated with intraplaque hemorrhage in basilar artery plaque

\begin{tabular}{|c|c|c|c|c|c|c|}
\hline \multirow[b]{2}{*}{ Age (years) } & \multicolumn{2}{|c|}{ Crude OR (95\% CI) } & \multirow{2}{*}{$\begin{array}{c}p \text { value } \\
0.066\end{array}$} & \multicolumn{2}{|c|}{ Adjusted OR (95\% CI) } & \multirow{2}{*}{$\begin{array}{r}p \text { value } \\
0.097\end{array}$} \\
\hline & 1.06 & $0.99-1.13$ & & 1.06 & $0.99-1.13$ & \\
\hline Male & 0.31 & $0.09-1.05$ & 0.060 & 0.31 & $0.08-1.21$ & 0.092 \\
\hline Hypertension & 4.73 & $0.56-40.12$ & 0.155 & & & \\
\hline Diabetes mellitus & 0.84 & $0.19-3.58$ & 0.817 & & & \\
\hline Hyperlipidemia & 4.53 & $1.22-16.80$ & 0.024 & 4.99 & $1.22-20.51$ & 0.026 \\
\hline Alcohol drinking & 0.55 & $0.13-2.26$ & 0.403 & & & \\
\hline Smoking & 0.33 & $0.07-1.69$ & 0.184 & & & \\
\hline
\end{tabular}

Results are expressed as odds ratio and $95 \%$ confidence interval. Variables with $p<0.1$ by univariate analysis were entered into the multivariate analysis model. CI, confidence interval.

Table 3. Univariate and multivariate analyses of the parameters associated with basilar artery dissection

\begin{tabular}{|c|c|c|c|c|c|c|}
\hline \multirow[b]{2}{*}{ Age (years) } & \multicolumn{2}{|c|}{ Crude OR (95\% CI) } & \multirow{2}{*}{$\frac{p \text { value }}{0.006}$} & \multicolumn{2}{|c|}{ Adjusted OR (95\% CI) } & \multirow{2}{*}{$\frac{p \text { value }}{0.027}$} \\
\hline & 0.93 & $0.89-0.98$ & & 0.94 & $0.89-0.99$ & \\
\hline Male & 1.12 & $0.30-4.16$ & 0.869 & & & \\
\hline Hypertension & 0.32 & $0.09-1.07$ & 0.111 & & & \\
\hline Diabetes mellitus & 0.48 & $0.09-2.44$ & 0.373 & & & \\
\hline Hyperlipidemia & 0.28 & $0.06-1.39$ & 0.120 & & & \\
\hline Alcohol drinking & 4.00 & $1.16-13.82$ & 0.028 & 2.38 & $0.61-9.21$ & 0.211 \\
\hline Smoking & 2.29 & $0.69-7.50$ & 0.173 & & & \\
\hline
\end{tabular}

Results are expressed as odds ratio and $95 \%$ confidence interval. Variables with $p<0.1$ by univariate analysis were entered into the multivariate analysis model. CI, confidence interval.

years old for BA plaque with IPH $[p<0.001])$. When the risk factors of atherosclerosis were compared, patients with BA plaque with IPH had the highest prevalence of hypertension ( $46.7 \%$ versus $73.3 \%$ versus $92.9 \%$, respectively, $p=0.021)$ and hyperlipidemia $(13.3 \%$ versus $35.6 \%$ versus $64.3 \%$, respectively, $p=0.017)$, whereas patients with BA dissection had the highest frequency of alcohol drinking $(66.7 \%$ versus $33.3 \%$ versus $21.4 \%$, respectively, $p=0.026)$.

Multivariate analysis was performed on factors associated with the presence of IPH within BA plaque after excluding patients with arterialBA dissection. The results confirmed that prevalence of hyperlipidemia was significantly different between patients with IPH and those without (odds ratio [OR], 4.99 [1.22-20.51]; $p=0.026$; Table 2). Another multivariate analysis was performed to compare patients with BA plaque without IPH and patients with BA dissection, and young age was significantly associated with BA dissection (OR, 0.94 [0.89-0.99]; $p=0.027$; Table 3).

\section{Discussion}

In this study, we performed HR-MRI on patients who distinctively had BA stenosis on TOF-MRA.
Afterward, BA stenosis patients were divided into the atherosclerotic plaque and dissection groups, and patients' characteristics were compared. The results showed that patients with BA plaque with IPH were the oldest, and hyperlipidemia was the most important factor for the development of IPH. On the contrary, patients with dissection were the youngest and had the highest alcohol drinking rate. However, the results of multivariate analysis indicated that age was the only significant factor.

IPH is highly associated with plaque progression. As the plaque progresses, the fibrous cap covering it becomes thinner and causes active inflammation. Inflammatory factors such as macrophages accumulate within the plaque and lead to ischemic stroke when it ruptures. Therefore, identifying IPH is critical to prevent future cerebrovascular events. IPH can be identified using T1-weighted MR sequences and TOF-MRA, but their accuracies are not high ${ }^{2,13)}$. Advanced MR sequences such as heavily T1-weighted techniques including MPRAGE and simultaneous non-contrast angiography and intraplaque hemorrhage (IPH) provide better accuracies ${ }^{2,14)}$. Basi-parallel anatomical scanning (BPAS) is a methodology for identifying the outer contour of the vertebro-BAs by using the fast spine-echo sequence ${ }^{15)}$. It is a relatively simple method by using T2-weighted imaging, and

\section{Advance Publication Journal of Atherosclerosis and Thrombosis}


the results of this test can be obtained relatively quickly (within two minutes). Conventional vascular examination methods are limited in evaluating the overall condition of the vessel wall because they only examine the inner contour. If BPAS is additionally used for diagnosing a vascular disease, arterial dissection can be detected more easily ${ }^{16)}$. Highresolution MRI takes longer than the BPAS method and it uses a contrast agent. However, it is expected that it can increase the diagnostic accuracy because it is possible to identify the internal condition of vessel contour as well as the inner and outer contours of the vessel.

It is generally known that the prevalence of carotid plaque with IPH is between $40 \%$ and $49 \%{ }^{13)}$. However, several recent studies using HR-MRI have reported that the ratio of IPH observed in BA highgrade stenosis cases is relatively low $(16 \%-21 \%)^{4,17)}$. Similarly, the prevalence of IPH in this study was $23.7 \%(14 / 59)$, which is lower than the general prevalence of IPH. Because the diameter of the BA is significantly small, the authors thought that IPH was detected only after the volume of IPH exceeded a certain threshold.

Additionally, the prevalence of a necrotic core was $16.9 \%$ in this study, which is similar to a previously reported rate ${ }^{18)}$. When the endothelial cells in the blood vessel wall are damaged, the LDL cholesterol and white blood cells flow into the tunica intima. White blood cells engulf the oxidized LDL cholesterol to produce foam cells. Subsequently, the lipids and debris of foam cells accumulate to form a necrotic core. As the necrotic core progresses, neovascularization occurs within the plaque from the vasa vasorum and contributes to the plaque burden. Therefore, the necrotic core is likely to facilitate progressive plaques and complications such as $\mathrm{IPH}^{19)}$. Elevated LDL cholesterol can affect the pathologic progression of various blood vessels, including large arteries and microcirculation ${ }^{20)}$. It is possible to stop plaque progression if active treatments, such as statins, are administered to patients ${ }^{21)}$. Detecting plaques with necrotic cores or IPH is thus critical.

Regarding arterial dissection, conventional digital subtraction angiography is considered the gold standard for diagnosis and follow-up. However, it is invasive and does not often show the status of the arterial wall directly, and this limits its use in accurately diagnosing dissection ${ }^{6}$. However, recent studies have reported that it is possible to diagnose arterial dissection noninvasively because HR-MRI can show the direct delineation of the arterial wall ${ }^{22}$. In this study, patients with BA dissection consumed alcohol more frequently than patients with atherosclerotic plaque. Alcohol generally leads to abrupt changes in blood pressure, which may affect the blood vessels ${ }^{23)}$. Alcohol-related increase in blood pressure is associated with a catecholamine surge, which is associated with aortic or carotid dissection ${ }^{24,25)}$. The catecholamine surge mechanism represents a possible explanation for the high rates of alcohol drinking across patients with BA dissection. However, according to the results of multivariate analysis, age was the only factor significantly affecting BA dissection. This outcome may be due to the small sample size, and large-scale studies are required to confirm this result.

The results of this study are significant; BA stenosis was reverified by HR-MRI. The characteristics of atherosclerotic plaque and arterial dissection were identified. In the atherosclerotic plaque, further differentiation was conducted according to the presence of IPH. However, this study has several limitations. First, this study has the possibility of selection bias because data were collected and analyzed retrospectively. Second, this study did not have a control group (patients without BA stenosis) because additional HR-MRI was performed only for patients with BA stenosis on the TOF-MRA. Third, the overall sample size was small because only a few patients had BA stenosis. As a result, it was impossible to divide the patients with BA stenosis into the symptomatic and asymptomatic groups, and it was difficult to include several variables in the multivariate analysis. Fourth, we could not perform a quantitative analysis of the plaque burden because the lumen of the BAs was anatomically very narrow. Finally, considering that this was a cross-sectional study, the status of IPH, or dissection through HR-MRI was not monitored over the long term. These limitations are expected to be resolved by future large-scale prospective and longterm monitoring studies.

\section{Conclusions}

This study found that $20 \%$ of patients diagnosed with BA stenosis using TOF-MRA had dissection after undergoing HR-MRI. Hyperlipidemia and young age significantly influenced the occurrence of IPH and dissection, respectively. Effective treatments can be administered when a precise stroke etiology is confirmed using HR-MRI. Therefore, the results of this study are expected to improve the prognosis of patients.

\section{Acknowledgements}

None. 


\section{Competing Interests}

The authors declare no conflicts of interest with respect to the research, authorship, funding, and/or publication of this article.

\section{Author Contributions}

HGK participated in the design and conceptualization of the study, performed the statistical analysis, interpreted the data, and drafted the manuscript. CHL and GHC contributed to the design and conceptualization of the study and revision of the manuscript for intellectual content. BSS and HSK contributed to the design and conceptualization of the study and interpretation of the data. HGK and HSK participated in the design and conceptualization of the study, performed the statistical analysis, interpreted the data, and revised the manuscript for intellectual content. All authors read and approved the final manuscript.

\section{References}

1) Connell L, Koerte IK, Laubender RP, Morhard D, Linn J, Becker HC, Reiser M, Brueckmann H and Ertl-Wagner B: Hyperdense basilar artery sign-a reliable sign of basilar artery occlusion. Neuroradiology, 2012; 54: 321-327

2) Yu JH, Kwak HS, Chung GH, Hwang SB, Park MS and Park SH: Association of Intraplaque Hemorrhage and Acute Infarction in Patients With Basilar Artery Plaque. Stroke, 2015; 46: 2768-2772

3) Takano K, Yamashita $S$, Takemoto $K$, Inoue T, Kuwabara $\mathrm{Y}$ and Yoshimitsu K: MRI of intracranial vertebral artery dissection: evaluation of intramural haematoma using a black blood, variable-flip-angle 3D turbo spin-echo sequence. Neuroradiology, 2013; 55: 845-851

4) Zhu C, Tian X, Degnan AJ, Shi Z, Zhang X, Chen L, Teng Z, Saloner D, Lu J and Liu Q: Clinical Significance of Intraplaque Hemorrhage in Low- and High-Grade Basilar Artery Stenosis on High-Resolution MRI. AJNR Am J Neuroradiol, 2018; 39: 1286-1292

5) Kim BM, Kim SH, Kim DI, Shin YS, Suh SH, Kim DJ, Park SI, Park KY and Ahn SS: Outcomes and prognostic factors of intracranial unruptured vertebrobasilar artery dissection. Neurology, 2011; 76: 1735-1741

6) Han M, Rim NJ, Lee JS, Kim SY and Choi JW: Feasibility of high-resolution MR imaging for the diagnosis of intracranial vertebrobasilar artery dissection. Eur Radiol, 2014; 24: 3017-3024

7) Kwak HS, Hwang SB, Chung GH and Jeong SK: Highresolution magnetic resonance imaging of symptomatic middle cerebral artery dissection. J Stroke Cerebrovasc Dis, 2014; 23: 550-553

8) Grundy SM, Stone NJ, Bailey AL, Beam C, Birtcher KK, Blumenthal RS, Braun LT, de Ferranti S, Faiella-Tommasino J, Forman DE, Goldberg R, Heidenreich PA, Hlatky MA, Jones DW, Lloyd-Jones D, Lopez-Pajares N, Ndu- mele CE, Orringer CE, Peralta CA, Saseen JJ, Smith SC, Jr., Sperling L, Virani SS and Yeboah J: 2018 AHA/ACC/ AACVPR/AAPA/ABC/ACPM/ADA/AGS/APhA/ASPC/ NLA/PCNA Guideline on the Management of Blood Cholesterol: A Report of the American College of Cardiology/American Heart Association Task Force on Clinical Practice Guidelines. Circulation, 2019; 139: e1082-e1143

9) Yamada N, Higashi M, Otsubo R, Sakuma T, Oyama N, Tanaka R, Iihara K, Naritomi H, Minematsu K and Naito $\mathrm{H}$ : Association between signal hyperintensity on T1-weighted MR imaging of carotid plaques and ipsilateral ischemic events. AJNR Am J Neuroradiol, 2007; 28: 287-292

10) Kwak HS, Yang HJ, Hwang SB and Chung GH: Carotid Wall Imaging with Routine Brain MRI to Facilitate Early Detection of Carotid Plaque and Intraplaque Hemorrhage. J Stroke, 2017; 19: 107-108

11) Naggara O, Louillet F, Touze E, Roy D, Leclerc $X$, Mas JL, Pruvo JP, Meder JF and Oppenheim C: Added value of high-resolution MR imaging in the diagnosis of vertebral artery dissection. AJNR Am J Neuroradiol, 2010; 31: $1707-1712$

12) Rodallec MH, Marteau V, Gerber S, Desmottes L and Zins M: Craniocervical arterial dissection: spectrum of imaging findings and differential diagnosis. Radiographics, 2008; 28: 1711-1728

13) Ota H, Yarnykh VL, Ferguson MS, Underhill HR, Demarco JK, Zhu DC, Oikawa M, Dong L, Zhao X, Collar A, Hatsukami TS and Yuan C: Carotid intraplaque hemorrhage imaging at 3.0-T MR imaging: comparison of the diagnostic performance of three T1-weighted sequences. Radiology, 2010; 254: 551-563

14) Zhang Q, Chen Z, Chen S, Liu X, Ning J, Han Y, Chen L, He L, Zhao X, Xiong Y, Guo H, Yuan C, Li R and Chen $\mathrm{H}$ : Angiographic contrast mechanism comparison between Simultaneous Non-contrast Angiography and intraPlaque hemorrhage (SNAP) sequence and Time of Flight (TOF) sequence for intracranial artery. Magn Reson Imaging, 2020; 66: 199-207

15) Nagahata M, Abe Y, Ono S, Hosoya T and Uno S: Surface appearance of the vertebrobasilar artery revealed on basiparallel anatomic scanning (BPAS)-MR imaging: its role for brain MR examination. AJNR Am J Neuroradiol, 2005; 26: 2508-2513

16) Fatima $Z$, Motosugi U, Okumura $A$, Ishigame $K$ and Araki T: Basi-parallel anatomical scanning (BPAS)-MRI can improve discrimination of vertebral artery dissection from atherosclerosis and hypoplasia. Acad Radiol, 2012; 19: 1362-1367

17) Huang B, Yang WQ, Liu XT, Liu HJ, Li PJ and Lu HK: Basilar artery atherosclerotic plaques distribution in symptomatic patients: a 3.0T high-resolution MRI study. Eur J Radiol, 2013; 82: e199-203

18) Lee YK, Kwak HS, Chung GH and Hwang SB: LipidRich Necrotic Core of Basilar Artery Atherosclerotic Plaque: Contrast-Enhanced Black Blood Imaging on Vessel Wall Imaging. Diagnostics (Basel), 2019; 9:

19) Sun J, Underhill HR, Hippe DS, Xue Y, Yuan C and Hatsukami TS: Sustained acceleration in carotid atherosclerotic plaque progression with intraplaque hemorrhage: a long-term time course study. JACC Cardiovasc Imaging, 
2012; 5: 798-804

20) Todate Y, Uwano I, Yashiro S, Chida A, Hasegawa Y, Oda T, Nagasawa K, Honma $H$, Sasaki $M$ and Ishigaki $Y$ : High Prevalence of Cerebral Small Vessel Disease on 7T Magnetic Resonance Imaging in Familial Hypercholesterolemia. J Atheroscler Thromb, 2019; 26: 1045-1053

21) Migrino RQ, Bowers M, Harmann L, Prost R and LaDisa JF, Jr.: Carotid plaque regression following 6-month statin therapy assessed by $3 \mathrm{~T}$ cardiovascular magnetic resonance: comparison with ultrasound intima media thickness. J Cardiovasc Magn Reson, 2011; 13: 37

22) Oppenheim C, Naggara O, Touze E, Lacour JC, Schmitt E, Bonneville F, Crozier S, Guegan-Massardier E, Gerardin E, Leclerc X, Neau JP, Sirol M, Toussaint JF, Mas JL and Meder JF: High-resolution MR imaging of the cervical arterial wall: what the radiologist needs to know. Radiographics, 2009; 29: 1413-1431

23) Goran KP: Excessive alcohol consumption and aortic dissection: probable but unexplored relation. Am J Emerg Med, 2009; 27: 1163-1165

24) Stewart SH, Oroszi G, Randall PK and Anton RF: COMT genotype influences the effect of alcohol on blood pressure: results from the COMBINE study. Am J Hypertens, 2009; 22: 87-91

25) Goran KP: Pheochromocytomas in aortic dissection patients: have they been missing or missed? Am J Emerg Med, 2008; 26: 626-627 